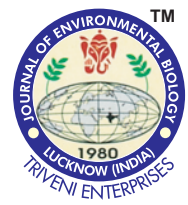

\title{
Expression of nitric oxide synthase isoforms in lacrimal glands of rats
}

\begin{abstract}
Authors Info
D.K. Park, Y. H. Yu, J.H. Kang, K.H. Lee, D.Y. Yoo, K.H. Park and D.S. Kim*

Department of Anatomy, Soonchunhyang University, Cheonan, 31151, Republic of Korea

*Corresponding Author Email : dskim@sch.ac.kr

\section{Edited by \\ Professor Seongho Ryu}

Reviewed by

Professor Ji Hyeon Ahn

Professor Jin Young Chung

\section{Abstract}

Aim: The present study was conducted to investigate the distribution of nitric oxide synthase isoforms (endothelial, neuronal, and inducible NOS) and changes in endothelial NOS (eNOS) expression after intraperitoneal injection of N(G)-Nitro-L-arginine methyl ester (L-NAME) in rats.

Methodology: Immunohistological and immunoelectron microscopic study, using monoclonal mouse antiendothelial NOS, anti-neuronal NOS and anti-inducible NOS were performed on the exorbital lacrimal glands of rats.

Results: eNOS-positive immunoreactivities were observed in the intralobular ducts and interlobular ducts of exorbital lacrimal gland of rats. One day after L-NAME administration, the size of eNOS-positive immunoreactivities had significantly decreased, however, after 3 and 5 days of L-NAME administration, eNOS-positive immunoreactivities were restored. Neuronal NOS-positive immunoreactivity was observed in nerve fibers of exorbital lacrimal gland, but was not detected in the duct systems or in the acinar cells. The inducible NOS was not detected in the exorbital lacrimal glands of rats.

Interpretation: Expression of nNOS in the nerves of lacrimal gland suggests that NO may play a role in modulating tear production. eNOS and nNOS were expressed in the exorbital lacrimal glands of rats, and the endogenously located eNOS was influenced by NOS inhibitor (L-NAME). These results suggest that endogenous NOS may be closely related with the direct or indirect regulation of glandular secretion and blood flow in the exorbital lacrimal glands.

Key words: Exorbital lacrimal gland, $\mathrm{N}$ w- Nitro-L-arginine methyl ester hydrochloride, Nitric oxide synthase, Tear production

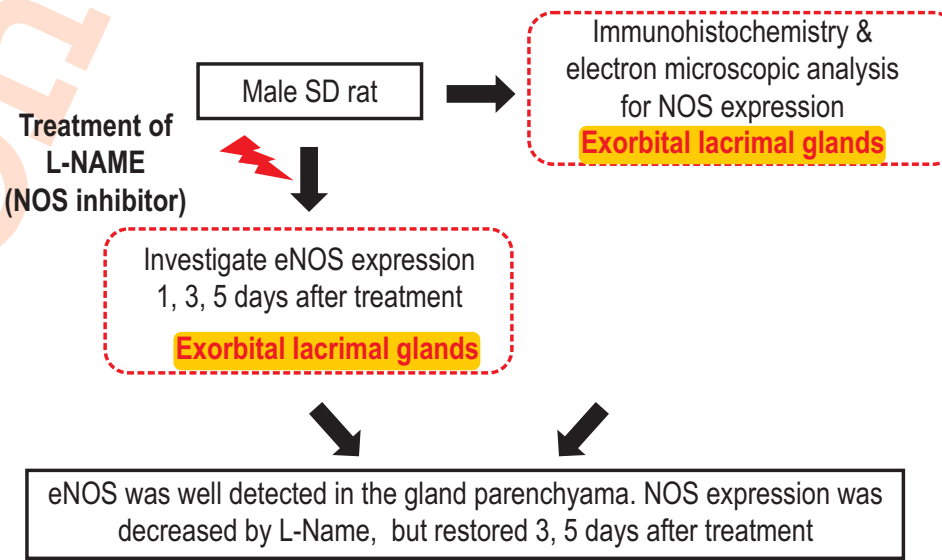

How to cite : Park, D.K., Y. H. Yu, J.H. Kang, K.H. Lee, D.Y. Yoo, K.H. Park and D.S. Kim: Expression of nitric oxide synthase isoforms in lacrimal glands of rats. J. Environ. Biol., 40, 847-853 (2019).DOI : http://doi.org/10.22438/jeb/40/5(SI)/SI-04
\end{abstract}




\section{Introduction}

Tears protect the eye ball from the invasion of foreign materials, and form three layers of precorneal film (Prydal et al., 1992). The mucin layer, which is the deepest layer, is produced by conjunctival goblet cells, while the middle aqueous layer consists of watery lacrimal fluid secreted from lacrimal glands. The most superficial layer is the lipid layer, which is formed by the tarsal glands in the eyelids. The aqueous and lipid layers prevent evaporation of mucin layer. An insufficient lipid layer or dysfunction of lacrimal glands cause dry eye syndrome, which consequently induces associated symptoms of the eyeballs and blurred vision (Beauregard and Brandt, 2004; Kanellopoulos and Asimellis, 2016). Inflammatory damage induces dysfunction of the lacrimal glands, and chronic irritation to lacrimal glands increases the production of nitric oxide (NO), which also causes inflammatory damage and cytotoxicity (Konttinen et al., 1997; Zoukhri, 2006). On the other hand, it has been reported that NO has protective role in various physiological and pathological situations (Becquet et al., 1997; Wink et al., 1996), therefore, the effects of NO in lacrimal glands need to be examined. The down regulation of $\mathrm{NO}$ by sex steroid regulates the immune response in the lacrimal glands (Beauregard and Brandt, 2004), implying the need for studies on the expressional changes of nitric oxide synthase (NOS) via NOS inhibitors to identify the causes of lacrimal gland associated diseases such as dry eye syndrome.

NO is known to not only regulate blood supply but also induce secretion of glands. NO triggers acetylcholine and vasoactive intestinal polypeptide in the parasympathetic nerves, and these factors induce vasodilation and regulation of blood supply (Edwards and Garrett, 1993; Edwards et al., 1996). In addition, it has been reported that endogenous NOS regulates secretion by directly acting on the acinar cells of glands (Buckle et al., 1995).

NOS has constitutive and inducible types, whereby the constitutive type consists of endothelial NOS (eNOS) and neuronal NOS (nNOS), while the inducible type consists of inducible NOS (iNOS). The constitutive NOS is made of calciumcalmodulin-dependent enzymes; eNOS and nNOS are, thus, regulated by intracellular calcium concentration. iNOS is a calcium-insensitive enzyme, and its expression is induced through the immune response stimulated by endotoxins and cytokines (Green et al., 1994; Knowles and Moncada, 1994).

Several studies have reported NOS expression in various glands (Craps et al., 2014; Zaragozá et al., 2010), however, no microscopic study has been carried out on the expression changes of NOS after the administration of $\mathrm{N} \omega$ - Nitro-L-arginine methyl ester hydrochloride (L-NAME), one of the NOS inhibitors, in lacrimal glands of rats. Many researchers have studied the NO synthesis and role of NOS with various NOS inhibitors in mammals (Costa et al., 2016; Poulos and Li, 2017). Among the
NOS inhibitors, N(G)-Nitro-L-arginine methyl ester (L-NAME) is known to act on all the NOS isoenzymes and is widely used (Knowles and Moncada, 1994).

In view of the above, in the present study, we investigated the expression of eNOS, nNOS and iNOS in lacrimal glands of rats, and confirmed the expression changes of eNOS after treatment of L-NAME through immunohistochemistry.

\section{Materials and Methods}

Experimental animals: Male Sprague-Dawley rats, approximately twenty in number and weighing about $200 \mathrm{~g}$, were purchased from OrientBio (Seongnam, South Korea) and housed in a conventional state under standard conditions (light/dark cycle $12: 12$, and $22 \pm 2{ }^{\circ} \mathrm{C}, 55 \pm 5 \%$ ). The care of animals was in accordance with current international policies (NIH Publication No. 85-23, 1985, revised 1996) and the entirety of the study was conducted with approval from the Laboratory Animal Care of Soonchunhyang University.

Experimental groups and drug treatment: The animals were divided into 4 groups: Control group; L-NAME (50 mg)-treated group sacrificed 1 day after treatment (L-NAME-1d); L-NAMEtreated group sacrificed 3 days after treatment (L-NAME-3d) and L-NAME-treated group sacrificed 5 days after treatment (LNAME-5d). L-NAME saturated in saline $\left(50 \mathrm{mg}^{10 \mathrm{ml}^{-1}}\right)$ was administered intraperitoneally and the rats in the L-NAME-treated groups were sacrificed 1,3 , and 5 days after L-NAME administration.

Tissue processing for histology: For histological analysis, the SD rats were anesthetized with urethane $\left(2 \mathrm{~g} \mathrm{~kg}^{-1}\right.$, Sigma-Aldrich; Darmstadt, Germany) and perfused transcardially with phosphate buffered saline (PBS; NaCl: $137 \mathrm{mM}, \mathrm{KCl}: 2.7 \mathrm{mM}$, $\mathrm{Na}_{2} \mathrm{HPO} 4: 4.3 \mathrm{mM}, \mathrm{KH}_{2} \mathrm{PO} 4: 1.4 \mathrm{mM}$ ), followed by $4 \%$ paraformaldehyde (Merck; Kenilworth, NJ) in PBS. Both exorbital lacrimal glands were removed and postfixed in $4 \%$ paraformaldehyde for $24 \mathrm{hrs}$ at $4{ }^{\circ} \mathrm{C}$. Following post-fixation, the sublingual glands were washed in phosphate buffered saline and dehydrated with graded concentrations of alcohol. The tissues were embedded in paraffin and sectioned using a microtome (Leica Microsystems $\mathrm{GmbH}$, Wetzlar, Germany) into $5 \mu \mathrm{m}$ sections.

Immunohistochemistry for nitric oxide synthase expression: The sections from all the groups were processed under similar conditions to ensure that immunohistochemistry data were comparable between the groups. The sections were deparaffinized and hydrated, then treated with $3 \%$ hydrogen peroxide in $60 \%$ methanol for $1 \mathrm{hr}$ to remove the activity of endogenous peroxidase. After washing in PBS, the sections were incubated in 10\% normal ovine serum in PBS for $1 \mathrm{hr}$. The sections were then incubated with primary antibodies in PBS with $0.3 \%$ bovine serum albumin (BSA) overnight at room 
temperature: mouse anti-endothelial nitric oxide synthase (eNOS, diluted 1:200, Transduction Laboratories; Lexington, $\mathrm{KY}$ ), mouse anti-neuronal nitric oxide synthase (nNOS, diluted 1:150, Transduction Laboratories) and mouse anti-inducible nitric oxide synthase (iNOS, diluted 1:150, Transduction Laboratories). The sections were washed three times with PBS, and sequentially incubated with biotinylated goat anti-mouse $\lg G$ (diluted 1:150, Vector Laboratories, Burlingame, CA) and streptavidin peroxidase complex (Vector Laboratories).The sections were visualized with 3, 3-diaminobenzidine tetrahydrochloride (Sigma-Aldrich) in $0.1 \mathrm{M}$ Tris- $\mathrm{HCl}$ buffer. Digital images were captured using a BX51 light microscope (Olympus Corporation, Tokyo, Japan) equipped with a digital camera (DP72; Olympus Corporation) connected to a computer monitor

Electron microscopic examination: For electron microscopic examination, exorbital lacrimal gland tissues were fixed in 2.5\% glutaraldehyde and $1.5 \%$ paraformaldehyde in $0.1 \mathrm{M}$ Millonig's phosphate buffer $(\mathrm{pH} 7.3)$. The tissues were post-fixed in $1 \%$ osmium tetroxide in the same buffer. Following post-fixation, the tissues were dehydrated with alcohol and propylene oxide, and embedded in araldite solution. Tissues were sectioned with ultramicrotome (LKB-V) into 60-70 nm- sections after staining with toluidine blue. Each section was mounted on a 200 mesh nickel grid and treated with sodium m-periodate for $1 \mathrm{hr}$, and then washed with distilled water 5 times. The sections were then incubated with 1\% BVA in tris buffered saline (TBS, pH 8.2) for 1 $\mathrm{hr}$, and monoclonal mouse anti-eNOS (diluted 1:150, Transduction Laboratories) for $1 \mathrm{hr}$ at room temperature for $20 \mathrm{hr}$ at $4{ }^{\circ} \mathrm{C}$ in sequence. After washing 5 times in TBS, the sections were incubated with $10 \mathrm{~nm}$ colloidal gold-goat anti-mouse IgG (diluted 1:20, Jackson Immuno Research Laboratory) for $1 \mathrm{hr}$ at room temperature. After immunogold staining, the grid was counterstained with uranyl acetate and lead citrate and observed under an electron microscope (JEOL, Model JEM 100CXII).

Statistical analysis: The data were expressed as mean \pm S.D. Differences between the means were analyzed by one-way analysis of variance, followed by Bonferroni's post hoc test. Analysis was performed using GraphPad Prism software (GraphPad Software, Inc., La Jolla), and significant differences were indicated if $p \leq 0.05$.

\section{Results and Discussion}

Expression of eNOS, nNOS and iNOS in the exorbitallacrimal glands: To investigate the expression pattern of NOS, the immunohistochemistry for eNOS, nNOS and iNOS was performed. In the lacrimal glands of SD rats, it was observed that gland parenchyma was covered by a capsule and the lobules were separated by connective tissues. Lobules consisted of serous glandular cells, and intercalated, intralobular and interlobular ducts were detected (Fig. 1A). eNOS-positive immunoreactivity was clearly detected in the intercalated, intralobular and interlobular ducts, but weak immunoreactivity for eNOS was observed in the vascular endothelial cells in the lobules of lacrimal glands (Figs. 1A, B). Immunoreactivity for eNOS was rarely detected in the glandular acini. nNOS-positive immunoreactivity was only detected in the nerve fibers in the interlobular connective tissues; however, no immunoreactivity for nNOS was detected in the interlobular and intralobular connective tissues (Fig. 1C). Immunoreactivity for iNOS was not detected in any of the subregions of lacrimal glands (Fig. 1D).

Few researchers have reported no detection of NOS expression in the vascular endothelial cells and secretory ducts via nicotinamide adenine dinucleotide phosphate staining (Alm et al., 1995; Modin et al., 1994), however, in the study eNOS expression was confirmed in the intercalated ducts, intralobular ducts and secretory granules of acinar cells by immunohistochemistry and electron microscopic examination. In the rat parotid gland, it was reported that NO plays a crucial role in salivary secretion, and endogenous nNOS exists in the acinar cells (Ishikawa et al., 2002). Hodges et al. (2005) reported that both eNOS and nNOS are expressed in the rat lacrimal glands, while eNOS exist in the acinar cells and nNOS in the nerve fibers of intra- and interlobular connective tissues (Hodges et al., 2005). Unlike previous reports, in this study, strong eNOS expression in the secretory ducts, and observed nNOS expression only in the nerve fibers of the interlobular connective tissues, not in the acinar cells. The expression of NOS and actions of NO are closely concerned with lacrimal secretion, and the pathological action of NO can cause oligodacrya (Bacman et al., 2001).

The exact role and mechanism of endogenous NOS have not been elucidated. We observed that endogenous NOS is expressed in the cytoplasm of intra- and interlobular duct cells, suggesting that NOS may have certain role in the secretory ducts, such as regulating the size of the ducts and duct secretion. Further studies on the secretory ducts of the glands are, therefore, essential in terms of endogenous NOS action.

Effects of L-NAME on eNOS expression: To investigate the effects of L-NAME (a non-selective NOS inhibitor) on eNOS, the eNOS expression was checked 1, 3 and 5 days after L-NAME administration. In the control group, it was observed that eNOSpositive immunoreactivity occurred mainly in the intercalated, intralobular and interlobular ducts. eNOS-positive immunoreactivity was rarely observed in the glandular acini (Fig. $2 A)$. In the L-NAME-1d group, eNOS expression was also detected in the same regions as those in the control group, but immunoreactivity for eNOS was signicantly decreased (Fig. 2B, E). In the L-NAME-3d and L-NAME-5d groups, immunoreactivity for eNOS was restored compared to that in the L-NAME-1d group (Figs. 2C,D, E). In the present study, it was confirmed that treatment with L-NAME inhibited the expression of eNOS transiently without microstructural changes of lacrimal glands. 

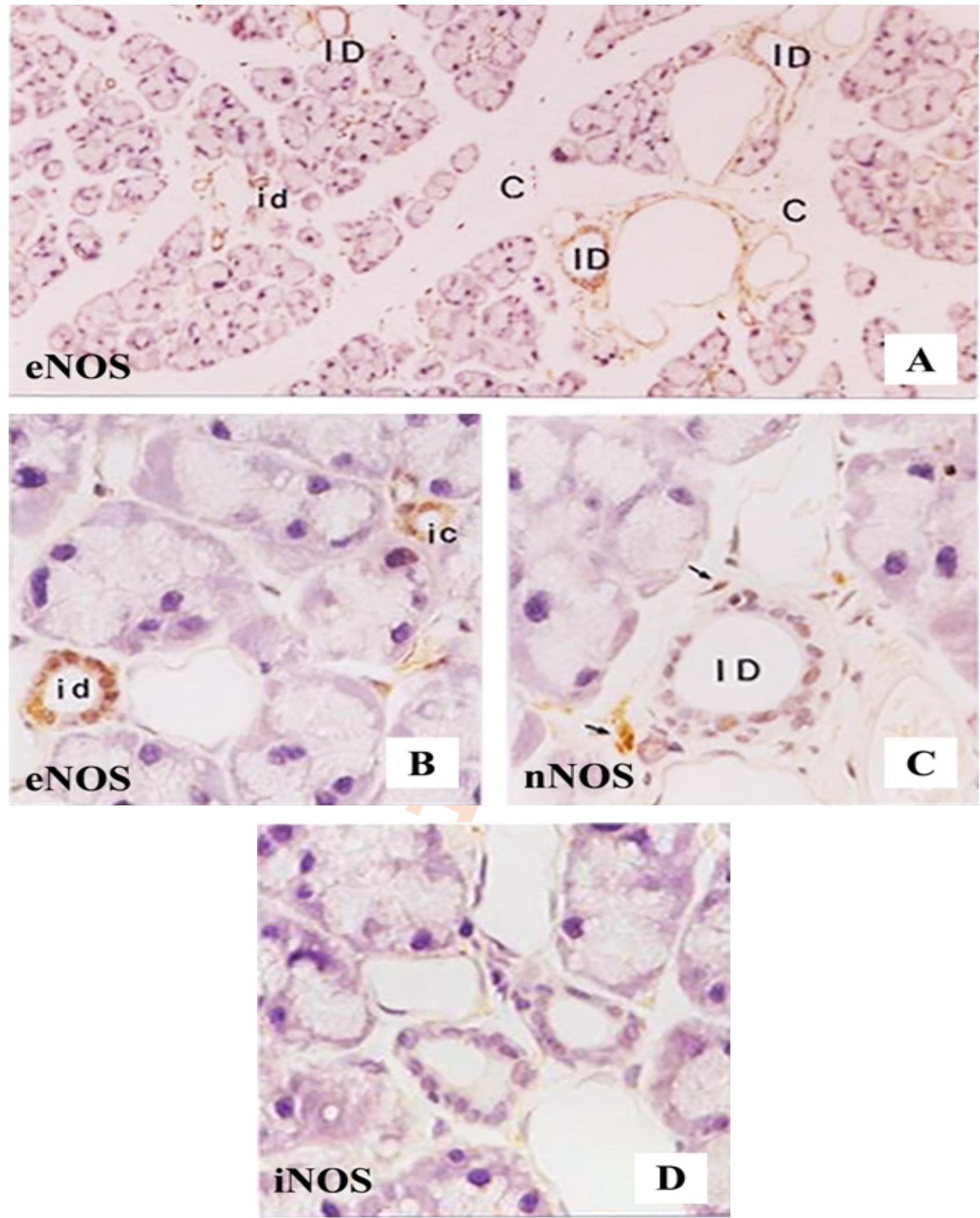

Fig. 1 : Immunohistochemistry for eNOS (A, B), nNOS (C), and iNOS (D) in the normal rat exorbital lacrimal glands. X100 (A), X400 (B, C, and D).eNOS immunoreactivity is well-detected in the secretory ducts $(A, B)$, but immunoreactivity for nNOS is detected only in the nerve fibers (arrows, C). iNOSpositive immunoreactivity is not detected (D). A: acinar cell; ID: interlobular duct. 

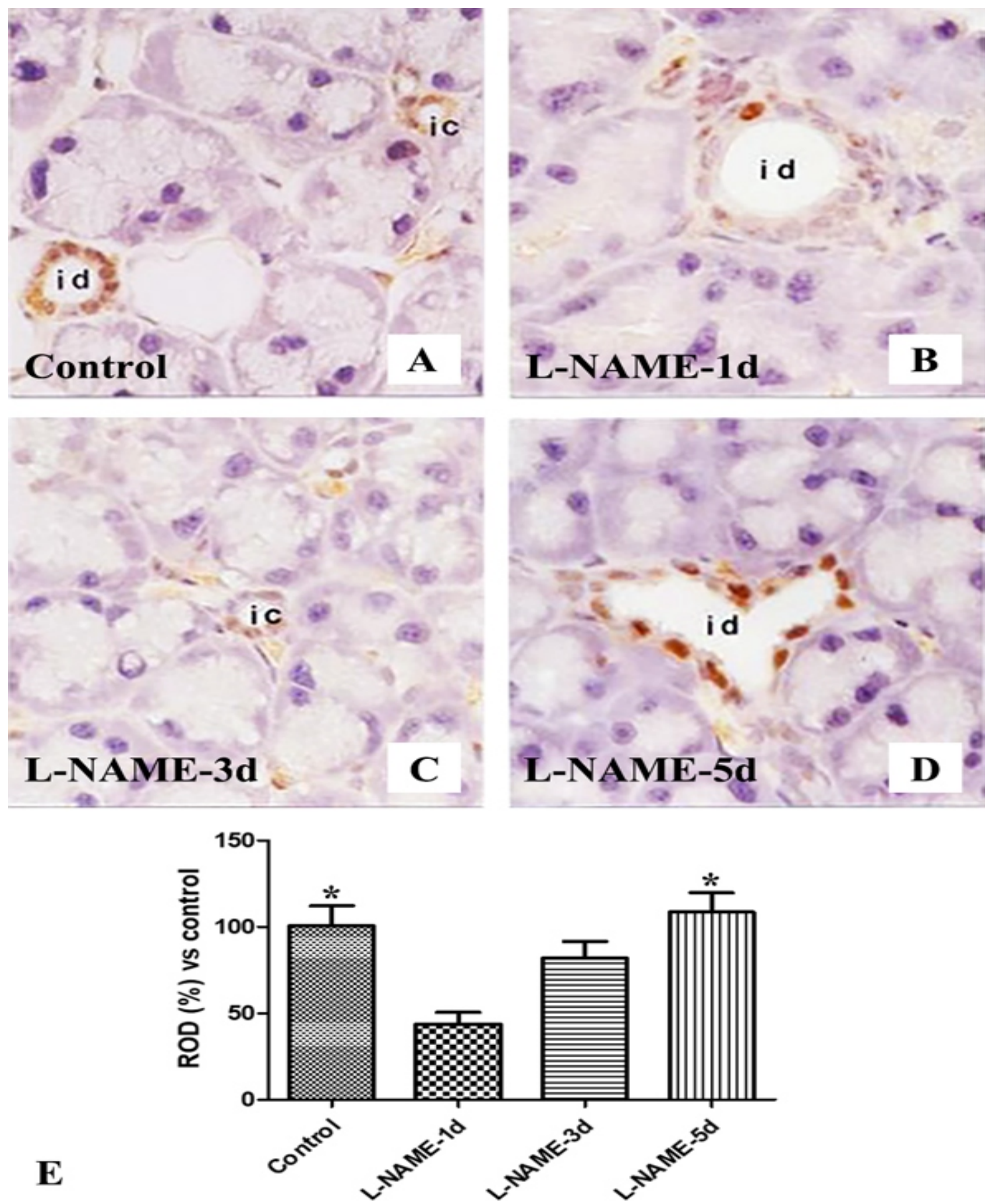

Fig. 2 : Immunohistochemistry for eNOS in the rat exorbital lacrimal glands of the control (A), L-NAME-1d (B), L-NAME-3d (C), and L-NAME-5d (D) groups. X400 (E) Relative optical density of eNOS-positive immunoreactivity compared to the L-NAME-1d group; * indicates a significant difference vs the L-NAME-1d group $(p<0.05)$. eNOS immunoreactivity is significantly decreased in the L-NAME-1d group, and restored in the L-NAME-3d and LNAME-5d groups. A, acinar cell; ic, intercalated duct; id, intralobular duct; ID: interlobular duct; ROD: relative optical density. 

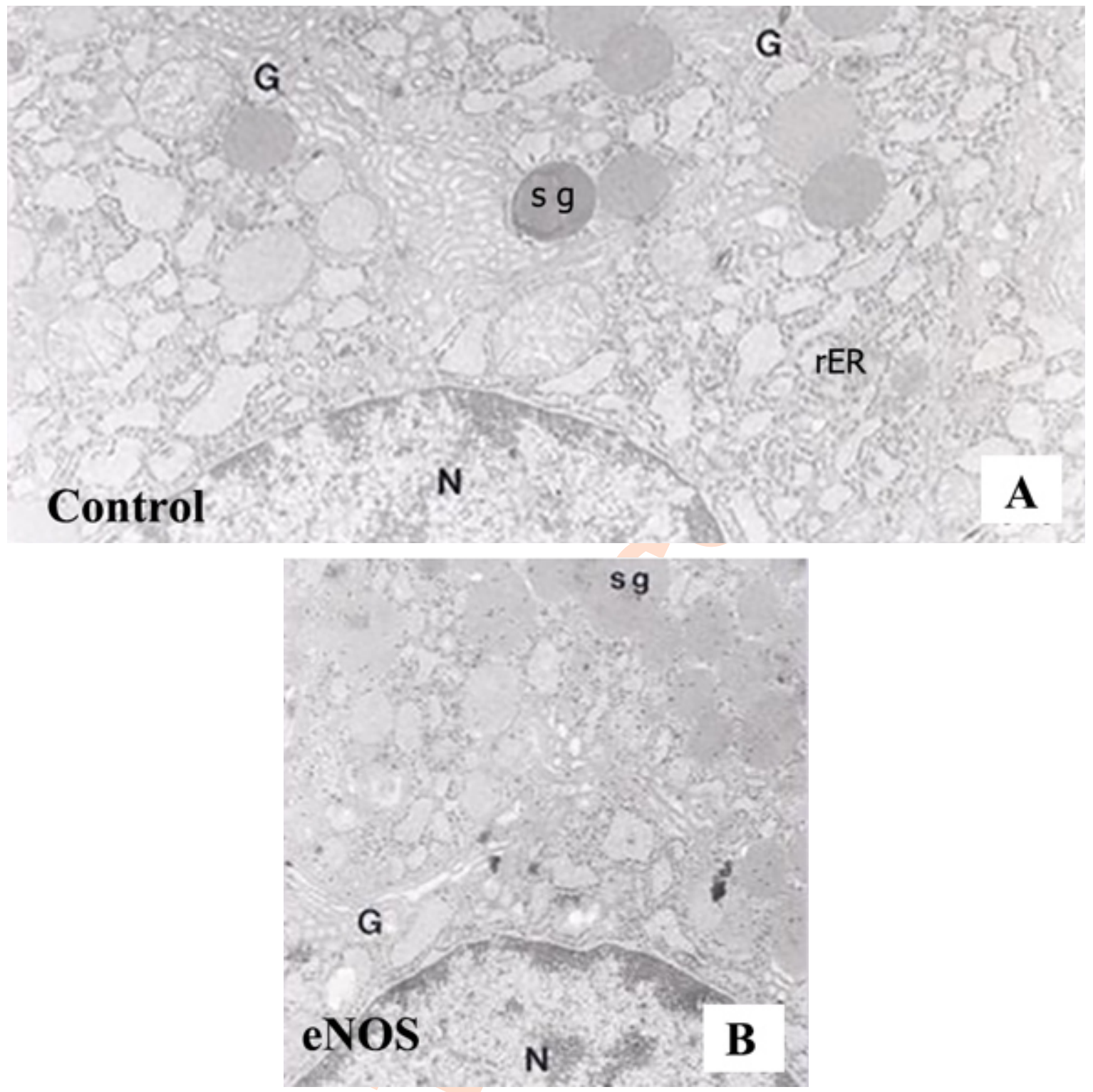

Fig. 3 : Electron micrograph of normal (A), and immunostained (eNOS, B) rat exorbital lacrimal gland. Golgi complexes are predominantly observed in the apical and middle portions of the acinar cell. The image shows the secretory granules (sg) of variable densities and sizes, and the well-developed rough surfaced endoplasmic reticulum (A). Many eNOS-positive gold particles are selectively located on the secretory granules (B). G, Golgi complex; N, nucleus; sg, secretory granule; rER, rough surfaced endoplasmic reticulum. Scale bar=1 $\mu \mathrm{m}$.

It was reported that treatment of NOS inhibitor in the rodent submandibular glands not only decreased the enzymatic activities of NOS by over $50 \%$ (Rosignoli et al., 2001), but also the secretion of saliva and proteins by $23 \%$ and $19 \%$ (Abdollahi and Safarhamidi, 2002). In the salivary glands, a decrease of eNOS expression was noted in the rat lacrimal glands after the administration of NOS inhibitor; these studies support that eNOS expression in the lacrimal glands is closely related to the regulation of lacrimal secretion.

eNOS expression in ultramicroscopic structures: To investigate ultramicroscopic structures and eNOS expression in the rat lacrimal glands, electron microscopic examination was performed. Under electron microscope, the golgi complexes were predominantly observed in the acinar cells. The secretory granules had variable density and size, and a well-developed rough surfaced endoplasmic reticulum was detected (Fig. 3A). In the acinar cell, eNOS-positive gold particles were well-detected and selectively located in the secretory granules (Fig. 3B).Confirmation of endogenous NOS in lacrimal glands supports that synthesized NO can regulate blood flow into lacrimal glands due to its size to cross cell membranes freely (Looms et al., 2002, Förstermann and Sessa, 2011). In addition, it has been reported that NO not only regulates blood flow, but also 
acts as a messenger molecule with various targets and also regulates gene transcription and post-translational modifications (Förstermann and Sessa, 2011; Toda et al., 2012).

In the present study, we confirmed that eNOS was clearly expressed in the secretary granules of acinar cells and intercalated ducts. The activities of eNOS were inhibited transiently after treatment of L-NAME, and were restored several days after treatment. These results suggest that NOS in the rat lacrimal glands is endogenous, and may be closely related to the regulation of production and secretion of tears.

\section{Acknowledgment}

This work was supported by Soonchunhyang University.

\section{References}

Abdollahi, M. and H. Safarhamidi: Protection by nitric oxide of morphineinduced inhibition of rat submandibular gland function. Pharmacol. Res., 45, 87-92 (2002).

Alm, P., B. Uvelius, J. Ekström, B. Holmqvist, B. Larsson and K.E. Andersson: Nitric oxide synthase-containing neurons in rat parasympathetic, sympathetic and sensory ganglia: A comparative study. Histochem. J., 27, 819-831 (1995).

Bacman, S., A. Berra, L. Sterin-Borda and E. Borda: Muscarinic acetylcholine receptor antibodies as a new marker of dry eye Sjogren syndrome. Invest. Ophthalmol. Vis. Sci., 42, 321-327 (2001).

Beauregard, C. and P. Brandt: Down regulation of interleukin-1 $\beta$-induced nitric oxide production in lacrimal gland acinar cells by sex steroids. Curr. Eye Res., 29, 59-66 (2004).

Becquet, F., Y. Courtois and O. Goureau: Nitric oxide in the eye: Multifaceted roles and diverse outcomes. Surv. Ophthalmol., 42, 71-82(1997)

Buckle, A.D., S. Parker, S. Bloom and A. Edwards: The role of nitric oxide in the control of protein secretion in the submandibular gland of the cat. Exp. Physiol., 80, 1019-1030 (1995).

Costa, E.D., B.A. Rezende, S.F. Cortes and V.S. Lemos: Neuronal nitric oxide synthase in vascular physiology and diseases. Front. Physiol., 7, 206 (2016)

Craps, J., C. Wilvers, V. Joris, B. De Jongh, J. Vanderstraeten, I. Lobysheva, J.L. Balligand, P. Sonveaux, P. Gilon and M.C. Many: Involvement of nitric oxide in iodine deficiency-induced microvascular remodeling in the thyroid gland: Role of nitric oxide synthase 3 and ryanodine receptors. Endocrinol., 156, 707-720 (2014).

Edwards, A. and J. Garrett: Nitric oxide-related vasodilator responses to parasympathetic stimulation of the submandibular gland in the cat.J. Physiol.,464, 379-392 (1993).

Edwards, A., G. Tobin, J. Ekstrom and S. Bloom: Nitric oxide and release of the peptide VIP from parasympathetic terminals in the submandibular gland of the anaesthetized cat. Exp. Physiol., 81, 349-359 (1996).

Förstermann, U. and W.C. Sessa: Nitric oxide synthases: Regulation and function. Eur. Heart J., 33, 829-837 (2011)

Green, S.J., L.F. Scheller, M.A. Marletta, M.C. Seguin, F.W. Klotz, M. Slayter, B.J. Nelson and C.A. Nacy: Nitric oxide: Cytokineregulation of nitric oxide in host resistance to intracellular pathogens. Immunol. Lett., 43, 87-94 (1994).

Hodges, R.R., M.A. Shatos, R.S. Tarko, J. Vrouvlianis, J. Gu and D.A. Dartt: Nitric oxide and cGMP mediate a1D-adrenergic receptor-stimulated protein secretion and p42/p44 MAPK activation in rat lacrimal gland. Invest. Ophthalmol. Vis. Sci., 46, 2781-2789 (2005).

Ishikawa, Y., H. lida, M.T. Skowronski and H. Ishida: Activation of endogenous nitric oxide synthase coupled with methacholineinduced exocytosis in rat parotid acinar cells. J. Pharmacol. Exp. Ther., 301, 355-363 (2002).

Kanellopoulos, A.J. and G. Asimellis: In pursuit of objective dry eye screening clinical techniques. Eye Vis., 3, 1 (2016).

Knowles, R.G. and S. Moncada: Nitric oxide synthases in mammals. Biochem. J., 298, 249-258 (1994).

Konttinen, Y.T., L.A. Platts, S. Tuominen, K.K. Eklund, N. Santavirta, J. Törnwall, T. Sorsa, M. Hukkanen and J.M. Polak: Role of nitric oxide in Sjögren's syndrome. Arthritis. Rheum., 40, 875-883 (1997).

Looms, D., K. Tritsaris, A.M. Pedersen, B. Nauntofte and S. Dissing: Nitric oxide signaling in saliavary glands. J. Oral Pathol. Med., 31, 569-584 (2002).

Modin, A., E. Weitzberg, T. Hökfelt and J. Lundberg: Nitric oxide synthase in the pig autonomic nervous system in relation to the influence of NG-nitro-L-arginine on sympathetic and parasympathetic vascular control in vivo. Neurosci., 62, 189-203 (1994).

Poulos, T.L. and H. Li: Nitric oxide synthase and structure-based inhibitor design. Nitric Oxide, 63, 68-77 (2017).

Prydal, J.I., P. Artal, H. Woon and F.W. Campbell: Study of human precorneal tear film thickness and structure using laser interferometry. Invest. Ophthalmol. Vis. Sci., 33, 2006-2011 (1992).

Rosignoli, F., N.B. Goren and C.P. Leirós: Alterations in nitric oxide synthase activity and expression in submandibular glands of NOD mice. Clin. Immunol., 101, 86-93 (2001).

Toda, N., K. Ayajiki and T. Okamura: Neurogenic and endothelial nitric oxide regulates blood circulation in lingual and other oral tissues. $\mathrm{J}$. Cardiovasc. Pharmacol.,60, 100-108 (2012).

Wink, D.A., I. Hanbauer, M.B. Grisham, F. Laval, R.W. Nims, J. Laval, J. Cook, R. Pacelli, J. Liebmann and M. Krishna: Chemical biology of nitric oxide: Regulation and protective and toxic mechanisms. Curr. Top. Cell. Regul., 34, 159-187 (1996).

Zaragozá, R., A. Bosch, C. García, J. Sandoval, E. Serna, L. Torres, E.R. García-Trevijano and J.R. Viña: Nitric oxide triggers mammary gland involution after weaning: Remodelling is delayed but not impaired in mice lacking inducible nitric oxide synthase. Biochem. J., 428, 451-462 (2010).

Zoukhri, D.: Effect of inflammation on lacrimal gland function. Exp. Eye Res., 82, 885-898 (2006). 